\title{
ANALISE BIOINFORMÁTICA PARA O DESENVOLVIMENTO DE INICIADORES PARA O GENE MATK DE ESPÉCIES DA FAMÍLIA ASTERACEAE VISANDO O CONTROLE DE QUALIDADE DE FITOTERAPICOS
}

\author{
Luiz Henrique da Silva Linhares ${ }^{1}$, Marília Alves de Lima ${ }^{1}$, Bruna Corina Silva de Lima ${ }^{1}$, Alice Conceição Moraes Florê \\ ncio ${ }^{1}$, Rodrigo Juan Martins Cardozo ${ }^{1}$, Claudia Sampaio Andrade Lima ${ }^{1}$, Ricardo Yara ${ }^{2}$ \\ ${ }^{1}$ Laboratório de Biofísica Química, UFPE \\ ${ }^{2}$ Departamento de Engenharia Biomédica, UFPE \\ ricardo.yara@gmail.com
}

\section{INTRODUÇÃO}

A utilização de plantas medicinais é uma prática que cresceu significantemente nos últimos anos, isto está relacionado com o resgate de ampla gama de conhecimentos a respeito das mesmas que foi acumulado por comunidades e grupos étnicos. Em regiões menos privilegiadas os tratamentos de enfermidades através do uso de plantas medicinais representam os únicos recursos para estas populações. Neste sentido, fitoterápicos produzidos a base dessas plantas são comercializados em feiras livres, mercados populares em diferentes regiões do país inclusive em grandes cidades (Maciel et al, 2001).

A venda destes produtos muitas vezes não possui certificado de qualidade e a produção é feita a partir de plantas não validadas, logo, as mesmas são consumidas com pouca ou nenhuma comprovação de suas propriedades farmacológicas. Além da possibilidade de intoxicação por plantas erroneamente identificadas e utilizadas que acaba se constituindo em um problema de saúde pública.

Os efeitos adversos das possíveis adulterações de lotes, contaminaç ões e toxidez são frequentes (Junior et al, 2005). Em geral isto ocorre devido a equívocos na identificação das plantas a serem utilizadas, uma vez que a nomenclatura popular de diversas plantas medicinais pode variar de acordo com as regiões do país.

A família Asteraceae é a maior dentre as Angiospermae, compreendendo aproximadamente 23 mil espécies, pertencentes a 1.535 gêneros, dispostos em 17 tribos (Juud et al., 2009). Sendo que muitas das espécies de Asteraceae são utilizadas como plantas medicinais. A espécie Acmella radicans, por exemplo, é bastante utilizada em tratamento de inflamações, infecções, febre, tosse, resfriado, disenteria e como analgésico para aliviar dores de dente (Rios-Chavez et al.,2003).

Nos estudos de sistemática molecular de plantas, o gene da maturase $\mathrm{K}$ (matK) tem se consolidado como um o principal gene para estudos taxonomicos e filogenéticos quando comparado a outros genes, como rbcL e trnT-F (Barthet e Hilu., 2007). Ademais, as informações de sequência do matK geram filogenias tão robustas quanto as construídas a partir de conjuntos de dados constituídos por outros genes combinados (Hilu et al., 2003).

Baseado na necessidade de identificar se os fitomedicamentos comercializados realmente possuem os extratos vegetais descristos, a utilização de ferramentas genéticas baseado no gene matK é interessante pois esta possui alta correlação filogenética com diversos taxons. Entretato, sendo este um gene relativamente grande, existe a dificuldade de detecção deste em produtos acabados devido a problemas de integralidade dos ácidos nucleícos destas amostras.

Neste sentido, iniciadores que possam realizar amplificações de trechos menores tem importância, já que poderão amplificar o DNA a partir de amostras degradadas, possibilitando o controle de qualidade e de rastreabilidade do produto.

0 objetivo do trabalho foi realizar análise de bioinformatica para desenvolver iniciadores que possam promover amplificações de trechos de DNA com até 500 pb da espécie Acmella radicans, e que também possam ser utilizados em outras espécies da familia Asteraceae.

\section{MATERIAIS E MÉTODOS}

Utilizando as sequências disponíveis no GenBank, foram realizadas duas análises de alinhamento de iniciadores, com auxílio da ferramenta BLAST - BASIC local alignment search tool, em espécies da família Astraceae.

A primeira análise foi para verificar quais iniciadores da literatura que melhor pareavam na sequência matK da Acmella radicans (GenBank AY215758.1), para que possam vir a realizar amplificaçõ es de trechos com no máximo 500 pares de bases. Para esta análise foram utilizando os iniciadores forward matK-490 (5'GAAATCTTGGTTCAAAYCCTTCG-3' Hilu et al., 2003), Reverse matK1483 (5'CCAAATACCAAATCCGWCCTCTA-3' Suwanchaikasam, et al., 2013), forward matik-390 (5'- CGATCTATTCATTCAATATTTC-3', forward matK-Kim1R 5'- ACCCAGTCCATCTGGAAATCTTGGTTC-3', reverse matK-Kim3F 5' -CGTACAGTACTTTTGTGTTTACGAG-3' Fazekas et al., 2012), forward matK-1 (5'ACTGTATCGCACTATGTATCA-3' Marx et al., 2010), e forward matKXF (5'TAATTTACGATCAATTCATTC-3' Forde et al., 2009).

Em seguida, utilizando a ferramenta para o desenvolvimento de novos iniciadores "primer-BLAST", foram projetados sete novos iniciadores para as áreas restantes da sequência, onde não havia cobertura de análise. Os iniciadores criados foram: forward matKGold (5'GAAGCGAGGGATTCGTCCAT-3'), reverse matK-Ace (5'GCCATGGGGCCTTATCCATT- ' 3 ), forward matK-Plus (5' GTCAATTTCAGGTTGGGTCG-3'), reverse matK-888 (5'GCTTGAAAAGCCCTGGCAAA -3'), forward matk-Jus (5'ATCGTTCAAGTGTGCGGCTA-3'), forward matK-Evo (5' CAGACTCCATTTTTGGGGCA-3'), e o reverse matK-Luck (5' ACCTTACCCAGCTCATCTGG-3'). 
Na segunda análise, dois iniciadores da primeira análise (forward 1 e reverse 1483) e os sete criados pelo Iniciador-BLAST, mais uma vez com auxílio da ferramenta BLAST, foram analisados para verificar em quais outras espécies da família Asteraceae, podem ser utilizados. Foram estudadas sequencias de Helianthus annus (GenBank AY215805.1); Acanthospermum australe (GenBank AY215757.1); Chromolaena odorota (GenBank KJ637187.1); Lactuca sativa (GenkBank GU109304.1); Solidago virgaurea (GenBank KP175127.1); Calendula officinalis (Genbank KM356075.1); Solidago litoralis (GenBank KP175130.1); Vernonia baldwinii (GenBank KT176587.1); Acanthospermum hispidum (GenBank FJ789804.1); Artemisia tridentata (GenBank AF456776.1); Solidago Canadensis (GenBank MF159464.1); e Artemisia vulgaris (GenBank JN894753.1).

Através da ferramenta computacional OligoanAlayzer foi realizado o último procedimento, onde foi verificado algumas informações específicas sobre os iniciadores, tais como Temperatura de Fusão, Peso Molecular, possibilidade de formação de Estrutura em Grampo e a temperatura de fusão que isso ocorre, possibilidade de formaçã o de homodímeros e heterodímeros etc.

\section{RESULTADOS E DISCUSSÃO}

$\mathrm{Na}$ primeira análise apenas dois dos iniciadores utilizados se parearam de forma adequada para seguir a proposta de delimitaçõ es de trechos com 500 pb, já que os outros ou não se parearam adequadamente ou foram identificados outros problemas como formação de heterodímeros, o que não ocasionaria amplificações. 0 forward 1 e o reverse 1483, foram os iniciadores que permaneceram e, juntos com os iniciadores criados no primerBlast, foi possível delimitar a sequência em sete trechos, onde nenhum deles ultrapassou o limite de $500 \mathrm{pb}$.

A cobertura do gene matk foi quase total, e ocasionou uma perda de $8 \mathrm{pb}$ entre sequencias, no início da sequência a perda foi de 27 $\mathrm{pb}$, antes do iniciador matK Forward Gold. Já no final da sequê ncia, após o iniciador matK Reverse 1483 , a perda foi de $81 \mathrm{pb}$. A figura 1 mostra detalhadamente onde cada iniciador se alinha na sequência matK da Acmella radicans.

$\mathrm{Na}$ figura 2 é mostrado a cobertura do gene dos prováveis produtos de amplificação (Figura 2A), bem como o tamanho do produto de amplificação esperado para cada trecho (Figura 2B).

Nas aplicações de PCR, a sequência do iniciador tem papel fundamental para que o ensaio ocorra de maneira eficaz. Por isso, a informação dos iniciadores obtidos através da análise foi importante para averiguar se os devidos pares irão conseguir realizar as amplificações dos trechos. É preciso, por exemplo, que a temperatura de anelamento do par de iniciadores seja semelhante, ausência de complementaridade entre as extensões 3' para que não ocorra formação de dímeros, evitar sequências palindrômicas e ter uma temperatura de fusão em torno de $50^{\circ} \mathrm{e}$ $90^{\circ} \mathrm{C}$. Na tabela 1 é mostrado algumas informações específicas de cada um dos iniciadores utilizados.

Tabela 1. Algumas informações dos iniciadores utilizados. Fonte: Linhares, 2017. \begin{tabular}{lllll}
\hline Iniciadores Qtd de bases T. de Fusão Peso M. Qtd de GC Qtd de E. em &
\end{tabular} G.

F. Gold

F. Fold

F. 1

F. Evo

F. Jus

R. Ace

R. 888

R. 1483

R. Luck

$\begin{array}{cc}20 & 57.1^{\circ} \mathrm{C} \\ 20 & 54.0^{\circ} \mathrm{C} \\ 21 & 50.6^{\circ} \mathrm{C} \\ 20 & 55.6^{\circ} \mathrm{C} \\ 20 & 56.9^{\circ} \mathrm{C} \\ 20 & 57.8^{\circ} \mathrm{C} \\ 20 & 56.9^{\circ} \mathrm{C} \\ 23 & 55.1{ }^{\circ} \mathrm{C} \\ 20 & 56.4^{\circ} \mathrm{C}\end{array}$

6

$>A Y 215758.1$ Acmella radicans maturase $\mathrm{K}$

(matR) gene, complete cds; chloroplast
CGGCTAGGATCTTTRACACATTTAGAT EAAGCGAGGGATTCGICCAPACCATCGGAAMGTTTGTA2GAC TrTaT7CTr CARTTCHGGITGGICGATTAATARATGGATARGGCCCCATGGCTTCAMTTAATTTCATTTTGATTH

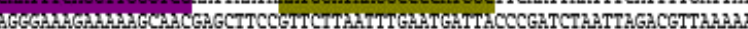
AAGATTAGTGCCCAMTACGGGALGCTTTCTCCCAGGARTGTATTCTTGATTTTTTAATGAATCCTAAM ATTACCACTCTCCATTCTATA2TGGAGAAGTATGTGTATAAGRAACAGTATATTGATA2AAACATTTCC2

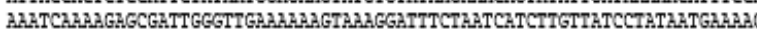

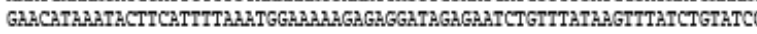
GAGGTATCTATTCGGTTGTACTATALTACCTTGITTIG CIGIAICGCACIAIGIAIC TITATALCCC

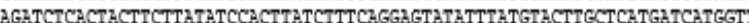

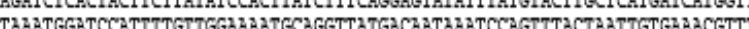
TA2ATGGATCCATTTTGTTGGLAATGCAGGTTATGACAATA2ATCCAGITTACTAATTGTGLACGTT2 AATCATTCGALTGIATCA2CAGAATCATTTGATCCTTTCTGITAATGATTCTA2ACAGACTCCATTTTT GGGCACAACAAGAATTTTTATTCGCA2GTAATGTCAGAGGTTTCTTCAACCATTRTGGAAATTCCATTGI CTCTGCGATTAMTATCTTCCCTAGA2AGGA2AGGGGTAGTTA2ATCCGATAMTTTACGATCAMTTCATT AATATTTTCTTTTTTAGAGGACAACTTTTCACATTTAALTTATGTATTAGATATACTAATACCTTZCCCR GCTCATCTGGA2ATCTTGGTTCAGGCTCTTCGCTATTGGATA2AAGATGCTTCCTCTTTGCATTTATTA GATTCTTTCTCCATGAGTGTCATAATTGGGATAGTCTTATTACTTCAAATTCA2AGAA2GCCAGTTCTTC TTTTTCAAA2AGA2ATCACAGACTATTCTTCTTCCTATATACTTCTTATGTATGTGALTATGAATCTGGC TTCCTCTTTCTACGTAACCAATCTTCTCACTTACGATCAACATCTTCTGGAGCCCTTATTGALCGA2TAT

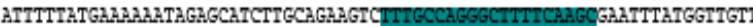
CA2AGATTCTTTCATGCATTATGTTAGGLATCA GGALAMTCAMTTCTIGCTTCAAALGGACGTTTCT

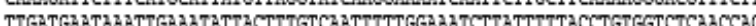
GLAGGATTTATATAAACCAATTATCCAMTCATTCCCTTGACTTTTTGGGTATCGITCARGIGTGCGEI

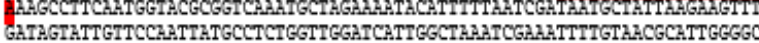
ATCCTATTGGTAAGGCGATTTGGGCCGATTTATCAGATTCTGATATTATTGAGCGCTTTGGCCGTATAT

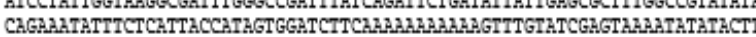
CGACTTTCTTGTGCTAGA2CTTTAGCTCGTAMGCATAM2AGTRCTGTACGTGCTTTTTTGAגAAGATTT

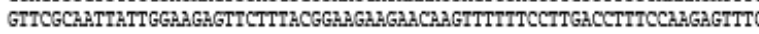
TICTATTTCGCGAAGGTITCTAGAAGGCGGATTTGGIATTTEGATATTGTTTGTATC2ATGATTTGGCC AATCATGAATGATTCGTTATGAAACGTTGTCAATCAAAACTTGATCACTAAATAT

\section{VERDE: FORWARD MATK GOLD \\ AMARELO ESCURO: REVERSE MATK ACE \\ VIOLETA:FORWRAD MATK PLUS \\ AZUL:FORWRAD MATK 1 \\ AMARELO: FORWARD MATK EVO \\ CINZA: REVERSE MATK LUCK \\ AZUL PETROLEO: REVERSE MATK 888 \\ VERMELHO: FORWRAD MATK JUS \\ TURQUESA: REVERSE MATK 1483}

Figura 1. Sequência matK da Acmella radicanis com a localização de cada iniciador. Fonte: os autores.

\section{A}

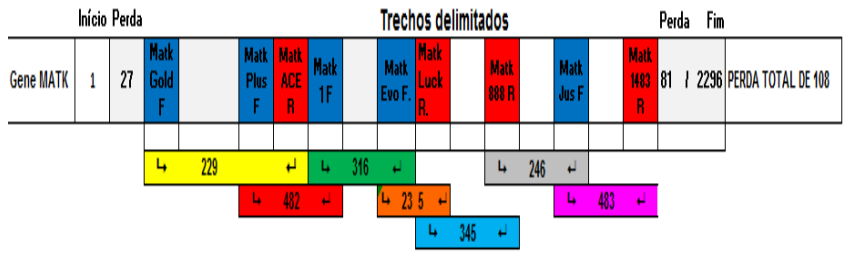

B

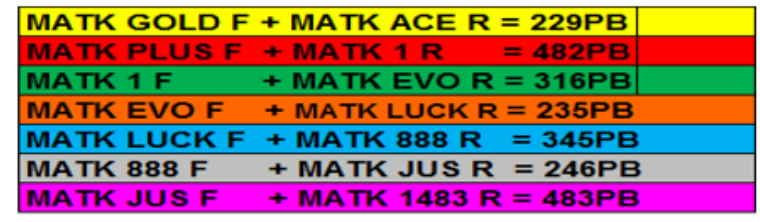

Figura 2. Características dos iniciadores e os possíveis produtos de amplificação (A) Imagem da localização dos paramentos dos iniciadores espacial da delimitação; (B) Tamanho esperados dos produtos de amplificação. Fonte: os autores. 
Os iniciadores puderam ser satisfatoriamente pareados com as sequências do gene matK das doze espécies da família Asteraceae analisadas. Onde algumas tiveram maior número de nucleotídeos pareados que outros.

Em todos os casos, a falha do pareamento não prejudicou em teoria a funcionalidade do iniciador. Na figura 3 é mostrado uma lista das espécies em que os iniciadores pareiam completamente. Nesta análise não foram consideradas a possibilidade de utilização de iniciadores degenerados, o que amplificaria o "range" de espé cies com pareamento completo.

\begin{tabular}{|c|c|c|c|c|c|c|c|}
\hline Matk Gold F T & MatkAce R. & 7 & Matk Plus F. & 7 & Matk $1 F_{\text {. }}$ & 7 & Matk EvoF. \\
\hline Soldagolthoral's & Acanthospermun autrale & & Solddgogolthoralis & & Solldggo litoral's & & Vernoneag badwinin \\
\hline Helanthus annus & & & Helianthus annus & & Soldidgovo virgogureg & & Acanthospermun australe \\
\hline Acranthospermun australe & & & Lactuca sativa & & Acanthospermun autrale & & Lactucos sativa \\
\hline Lactua sativg & & & Acranhospermun ausirale & & Helianthus arnus & & Acarthospermun hispidum \\
\hline \multirow[t]{2}{*}{ Calendula officinalis } & & & Caendula officinalls & & Lactuag saivg & & Hellanthus annus \\
\hline & & & & & Calendula officinalilis & & \\
\hline
\end{tabular}

\begin{tabular}{|r|c|c|c|}
\hline Matk Jus F. & Matk Luck R. & Matk 888 R. \\
\hline Acanthospermun australe & Chromolaena odorata & Chromolaena odorata & Solidago litoralis \\
\hline Artemisia tridentata & Helianthus annus & Helianthus annus & Helianthus annus \\
\hline Lactuca sativa & Acanthospermun australe & Acanthospermun australe & Vernonea baldwini \\
\hline Helianthus annus & Acanthospermun hispidum & Lactuca sativa & Chromolaena odorata \\
\hline Chromolaena odorata & Solidago virgaurea & Solidago litoralis & Acanthospermun australe \\
\hline Vernonea baldwinii & Vernonea baldwinii & Calendula officinalis & Lactuca sativa \\
\hline Solidago litoralis & Lactuca sativa & Vernonea baldwinii & Artemisia vulgaris \\
\hline Calendula officinalis & Artemisia tridentata & & \\
\hline
\end{tabular}

Figura 3. Lista de outras espécies da família Asteraceae que tiveram pareamento completo dos iniciadores com sequências do gene matK de diversas espécies. Fonte: os autores.

\section{CONCLUSÕES}

A partir das análises de pareamento e das informações obtidas dos iniciadores, há um forte indício de que haverá a obtenção de amplificações dos trechos. Uma vez que possam ser utilizados, esses iniciadores vão servir para identificação das espécies, atuando como um controle de qualidade dos fitomedicamentos.

\section{REFERÊNCIAS}

BARTHET, M. M.; HILU, K. W., 2007. Expression of matK: functional and evolutionary implications. American Journal of Botany 94: 1402-1412.

BLAST - BASIC local alignment search tool. Acesso em 20, Ago, 2017. Disponivel em: https: //blast.ncbi.nlm.nih.gov

FAZEKAS, A.J., KUZMINA, M.L., NEWMASTER, S.G., HOLLINGSWORTH, P.M. DNA Barcoding Methods for Land Plants W. John Kress and David L. Erickson (eds.), DNA Barcodes: Methods and Protocols, Methods in Molecular Biology, vol. 858, DOI 10.1007/978-1-61779-591-6_13, Springer Science Business Media, LLC 2012.

FORD CS, AYRES KL, HAIDER N, TOOMEY N, VAN-ALPEN-STOHL J, (2009) Selection of candidate DNA barcoding regions for use on land plants. Botanical Journal of the Linnean Society 159: 1-11.
HILU, K. W., T. BORSCH, K. MÜLLER, D. E. SOLTIS, P. S. SOLTIS, V. SAVOLAINEN, M.W. CHASE, M. P. POWELL, L. A. ALICE, R. EVANS, H. SAUQUET, C. NEINHUIS, T. A. B. SLOTTA, G. R. JENS, C. S. CAMPBELL, L. W. CHATROU. 2003. Angiosperm phylogeny based on matK sequence information. American Journal of Botany 90: 17581776.

Primer - Blast. Acesso em 15, Set, 2017. Disponível em: https://www.ncbi.nlm.nih.gov

JUDD, W.S., CAMPBELL, C.S., KELLOGG, E.A., STEVENS, P.F., DONOGHUE, M.J. 2009. Sistemática vegetal: um enfoque filogené tico. $3^{\text {a }}$ ed. Artmed, Porto Alegre. $612 \mathrm{p}$.

JUNIOR, V.F.V., PINTO, A.C., MACIEL, M.A.M., 2005. Plantas Medicinais: Cura segura? Quim. Nova, Vol. 28, No. 3, 519-528, 2005.

MACIEL, M.A.N., PINTO, A.C., JUNIOR, V.F.V., 2002. Plantas Medicinais: A necessidade de estudos multidisciplinares. Quim. Nova, Vol. 25, No. 3, 429-438, 2002.

MARX HE, O'LEARY N, YUAN Y-W, LU-IRVING P, TANK DC, MÚLGURA M, OLMSTEAD RG. 2010. A molecular phylogeny and classification of Verbenaceae. American Journal of Botany 97: 1647-1663.

OligoAnalyzer 3.1. Acesso em 15, Set, 2017. Disponível em: https://www.idtdna.com/calc/analyzer

Reverse Complement. Disponível em: https://www.bioinformatics.org/sms/rev_comp.html RIOS-CHAVEZ, P., RAMIREZ-CHAVEZ, E., ARMENTA, C. ; TORRES, M., J. Plant, 39, 2003, 37-41.

SUWANCHAIKASAM, P., PHADUNGCHAREON, T., SUKHADA, S., 2013. Authentication of the Thai medicinal plants sharing the same common name 'Rang Chuet': Thunbergia laurifolia, Crotalaria spectabilis, and Curcuma aff. amada by combined techniques of TLC, PCR-RFLP fingerprints and antioxidant activities. Science Asia. 39:124-133. 